\title{
Políticas públicas educativas y las sexualidades en Chile post-dictadura: opacidades e hiper-visibilidades de sujetos LGTBI
}

\section{Políticas educativas públicas e sexualidades no Chile pós-ditadura: opacidades e hipervisibilidades de sujeitos LGTBI}

\section{Public educational policies and sexualities in post-dictatorship Chile: opacities and hyper-visibilities of LGTBI subjects}

\author{
Caterine Galaz Valderrama* \\ Rolando Poblete Melis***
}

\begin{abstract}
RESUMEN
Una vez acabada la dictadura cívico-militar en Chile, progresivamente los distintos gobiernos comenzaron a prestar interés a la necesidad de una formación educativa a nivel sexual. No obstante, los enfoques que han dominado socialmente se relacionan con una transmisión heteronormativa de la sexualidad y, además, sujeta a marcos conservadores y una comprensión biologicista de las relaciones interpersonales. A partir de un análisis discursivo $^{1}$ del conjunto de leyes y directrices públicas en la educación entre los años 1990 y 2017, se visualiza cómo la diversidad sexual emerge como un
\end{abstract}

1 Esta investigación fue financiada por el proyecto FONDECYT N ${ }^{\circ} 1170417$ Acción pública y diversidad sexual en Chile: construcciones sociales en democracia (1990-2016).

*Universidad de Chile. Santiago, Chile. E-mail: cgalazvalderrama@uchile.cl.https://orcid. org/0000-0001-6301-7609.

** Facultad de Educación, Centro de Investigación en Educación. Universidad Bernardo O’Higgins. Santiago, Chile. E-mail: rolando.poblete@ubo.cl. https://orcid.org/0000-0002-7799-7190. 
constructo social que se pone en juego a partir de la opacidad/visibilidad de sus diferencias, y cómo se puede trazar la inclusión de derechos LGTBI en cuatro momentos históricos: uno inicial, con una política institucional del silencio sobre la temática; uno segundo, donde se instaura un marco general entorno a los programas educativos con alusiones generalistas sobre la orientación e identidad sexual, uno tercero, donde se evidencia una cierta disputa cultural con tintes moralistas y conservadores, y uno último, donde asistimos a una hipervisibilidad identitaria a respecto de sexualidades no heterosexuales.

Palabras clave: Diversidad sexual. LGTBI. Educación. Políticas públicas. Chile. Sexualidades. Educación sexual.

\title{
RESUMO
}

Uma vez que a ditadura civil-militar no Chile acabou, vários governos gradualmente começaram a desenvolver interesse à necessidade de formação educacional a nível sexual. No entanto, as abordagens que têm dominado se relacionam socialmente com uma transmissão de sexualidade heteronormativa, e também sujeita a estruturas conservadoras e compreensão biologicista das relações interpessoais. A partir de uma análise discursiva de todas as leis e diretrizes públicas de educação entre 1990 e 2017, mostra-se como a diversidade sexual surge como uma construção social que entra em jogo a partir da opacidade/visibilidade de suas diferenças e como a inclusão dos direitos LGTBI pode ser traçada em quatro momentos históricos: um inicial, com uma política institucional de silêncio sobre o assunto; um segundo, no qual um quadro geral que defina programas educacionais com alusões gerais à orientação sexual e identidade de gênero, um terceiro, no qual uma certa disputa cultural com tons moralistas e conservadores, e um último, no qual se assiste a uma hipervisibilidade identitária sobre sexualidades não heterossexuais.

Palavras-chave: Diversidade sexual. LGTBI. Educação. Políticas Públicas. Chile. Sexualidades. Educação Sexual.

\begin{abstract}
Once the civil-military dictatorship had come to its end in Chile, the following governments progressively began to pay attention to the need for an educational formation regarding sexuality. However, the predominant social approach to sexuality has been of a hetero-normative character, bound to the conservative spectrum and a biologic approach to interpersonal relations. Based on the discourse analysis of the set of laws and public guidelines on education in force between 1990 and 2017, it is possible to see how sexual
\end{abstract}


diversity emerges as a social construct that comes into play based on the secrecy/visibility of its differences. Also, the inclusion of LGTBI rights can be traced back to four historic moments: an initial one, when there was a policy of institutional silence on the topic; a second one, when a general framework is established for educational programs with generalist references regarding sexual orientation and identity; a third one, when it is possible to discern a certain cultural dispute based on moralist and conservative perspectives; and a final one, where hyper-visibility of non-heterosexual sexualities takes force.

Keywords: Sexual diversity. LGTBI. Education. Public policies. Chile. Sexualities. Sexual education.

\section{Introducción}

En las últimas décadas, la irrupción del debate de derechos LGTBI en la intervención pública en Chile parece ser un hecho relevante en tanto denota una renovada preocupación del Estado por unas dimensiones de la vida tradicionalmente relegadas al ámbito médico-jurídico (GONZÁLEZ; NÚÑEZ; GALAZ VALDERRAMA; TRONCOSO; MORRISON JARA, 2018). La intervención pública en diversidad sexual empieza a operar sobre una multiplicidad de sujetos, cuya condición los sitúa como objetos/sujetos, colectivos "vulnerables" o "minorías", susceptibles de intervenir, es decir gestionar mediante una serie de políticas identitarias orientadas a su inclusión. A partir del término de la dictadura chilena - período en que se discriminó abiertamente a personas LGBTI (ROBLES, 2008)- se ha dado cierta expansión de derechos, los cuales van desde legislaciones anti discriminación hasta el reconocimiento de la convivencia interpersonal. Los mayores desafíos y cambios relacionados con las luchas de estos colectivos se han ubicado en el ámbito de la salud y los derechos civiles, y particularmente en el campo de la educación (MOVILH, 2015).

Por lo tanto, este artículo busca reflexionar de qué manera esta irrupción temática de la diversidad sexual en el ámbito público en Chile, podría reificar en la educación, lógicas normativas, invisibilizando o hipervisibilizando diferencias, además de mantener y promover representaciones heteronormativas y etnocéntricas (VALENTINE, 2007; SABSAY, 2011). Asumimos que es necesario problematizar cómo se ha ido entretejiendo la incorporación de las sexualidades LGTBI en las intervenciones públicas en educación; precisamente para develar los discursos vinculados al ejercicio de la función estatal en esta materia y sus 
efectos en los colectivos LGTBI. Hasta la fecha no existe una política explícita que hable sobre educación en sexualidades no normativas, aunque sí se promulgaron en democracia algunas leyes y directrices ministeriales que dan cuenta sobre elementos de las sexualidades LGTBI, pero que revelan posiciones ideológico-valóricas de los gobiernos de turno. Asimismo, se han desarrollado programas y orientaciones sobre educación sexual, que intentaron instaurar alguna apertura pública sobre diversidad sexual. Pese a esto, la educación sexual no ha sido "explícitamente obligatoria dentro del ordenamiento jurídico chileno, quedando la implementación de los respectivos programas a merced de la libertad de enseñanza de cada escuela, tal como lo dispuso la Ley Orgánica Constitucional de Educación" (ARENAS; DURAN; DIDES; FERNÁNDEZ, 2016, p. 17). Planteamos que la evolución de la educación sexual en Chile y la inclusión de una perspectiva de derechos respecto de los colectivos LGTBI, se puede trazar en cuatro momentos: el primero, entre 1990-1995, caracterizado por el inicio de la institucionalización de la educación sexual y el silencio formal u opacidad respecto de la diversidad sexual; el segundo, entre 1995-2000, caracterizado por la participación del Ministerio de Educación que define un marco político general de referencia y emergencia de los primeros programas de sexualidad a nivel nacional donde la diversidad es tomada como un término "sombrilla" (CAREAGA, 2004), invisibilizando sus diferencias; el tercer periodo, desde 2000 a 2009, en que se crean diversos programas privados y públicos y se visualiza polarizaciones respecto a la concepción de la diversidad sexual y a los conceptos que le atañen a cada representación; y el cuarto periodo de 2010 a 2017, donde el Estado inicia una experiencia en educación sexual nacional a través de nuevas leyes, financiamientos de fondos centralizados y de orientaciones para las instituciones, en que se percibe cierto propósito de inclusión de una perspectiva de derechos LGTBI y como veremos más adelante, de cierta saturación identitaria (ROMERO y GARCÍA DAUDER, 2003).

Constituido el primer gobierno democrático, se planteó en 1991 una cierta inquietud por favorecer una educación sexual, sobre todo, vinculada a la lucha internacional contra el VIH. Se constituyó una comisión consultiva en Educación Sexual, que elaboró recomendaciones de política pública que consolidó la "Política de Educación en Sexualidad para el Mejoramiento de la Calidad de la Educación" (1993) con modificaciones hasta 2003. Ahí se evitó la imposición de contenidos educativos y definió diferentes criterios y procedimientos, dejando la responsabilidad de elaborar programas de educación sexual a las mismas escuelas. En ese sentido, se priorizó la autonomía y regulación de contenidos a partir de la ideología de los respectivos establecimientos educativos. La decisión política de que la educación sexual quedara como prerrogativa de cada centro tuvo su eco en el programa "Adolescencia, tiempo de decisiones" del Centro de 
Medicina Reproductiva y Desarrollo Integral de la Adolescencia (CEMERA). Éste fue publicado en 1998 (con re-ediciones en 2000 y 2002) y constituye uno de los primeros manuales para el profesorado, estudiantes y familias en educación sexual. Éste deja en claro su posición binarista y heteronormada: cada ser humano construye una identidad sexual basada en los roles de género, hombre y mujer, que socialmente establezca su cultura (CEMERA, 1993). Paralelamente, el Ministerio de Educación con el Ministerio de Salud, el Servicio nacional de la Mujer y el Instituto Nacional de la Juventud presentaron la propuesta de "Jornadas de Conversación sobre Afectividad y Sexualidad" (JOCAS, 1996; 2000). En ésta se describió a la sexualidad como una dimensión del ser humano que "compromete lo biológico, lo psicológico, lo social, lo ético y lo filosófico, e integra razón y conciencia; debe entenderse como sustantiva al ser mujer y al ser hombre" (JOCAS, 1999, p.23). En 2005, se generó una comisión de evaluación que entregó recomendaciones en las que se incluyeron la discriminación por orientación sexual. Sólo a partir de la promulgación del Plan nacional de Educación en Sexualidad y Afectividad (2005-2010), se comienzan a visibilizar explícitamente una apertura en torno a la sexualidad no heterosexual, principalmente homosexual (MINEDUC, 2005).

En 2009, se dicta la Ley 20.370, que establece la Ley General de Educación, donde se instauran normativas para el funcionamiento de los establecimientos educacionales. Las acciones apuntan a la no discriminación de niños, niñas y estudiantes, entre ellos/la, la población LGTBI. En 2010, se dicta la Ley 20.418 que fija normas sobre información, orientación y prestaciones en materia de regulación de la fertilidad. En esta ley aparecen nociones que rompen la lógica binaria, al introducir conceptos generales de diversidad sexual. En 2011 se reorienta la educación sexual, creando el Portafolio de Programas de Educación Sexual (2011). El Ministerio de Educación propone un listado de cursos sobre educación sexual, la mayoría establecidos bajo una lógica heterosexual. El año 2011 se dicta la Ley 20.536 sobre Violencia Escolar, que tiene por objetivo abordar la convivencia interpersonal, combatir el acoso escolar y prevenir la violencia escolar. A diferencia de otros países, esta ley no establece los tipos de violencia que serán afectadas por la normativa, como el acoso por orientación e identidad sexual. ${ }^{2}$ En 2013, MINEDUC propone el enfoque de Formación en Sexualidad, Afectividad y Género. A partir de este trabajo se diseña un programa en sexualidad, afectividad y género que funciona desde el año 2015. El documento se enfoca

2 Sólo a manera de ejemplo destacamos la legislatura de la ciudad Autónoma de Buenos Aires lo tipifica y sanciona a partir de la Ley 5.742 sobre acoso sexual en espacios públicos, lo que incluye la escuela, lo mismo la Ley Andaluza 2/2014 conocida como Ley Integral para la no discriminación por motivos de identidad de género. 
en el autocuidado y prevención, profundizando en los elementos constitutivos a la sexualidad, afectividad y género. Abiertamente asumen que la diversidad sexual ha estado teñida de "silencio, prejuicio, estereotipos que actúan como obstáculo cuando se piensa considerarlo como contenido o como parte de algún proyecto educativo que contemple su inclusión" (MINEDUC, 2015, p.12). Es importante señalar que, a partir de estas orientaciones, se plantean, conceptualizaciones explícitas sobre los significados de heterosexualidad, homosexualidad y bisexualidad, quedando en una zona más difusa la transexualidad e intersexualidad. Sólo en el año 2017 se establecen las "Orientaciones para la inclusión de las personas lesbianas, gays, bisexuales, trans e intersex" en el sistema educativo chileno ${ }^{3}$.

\section{Herramientas teóricas: educación y ciudadanías sexuales}

El cuestionamiento a las lógicas heteronormadas de la sexualidad ha constituido en las últimas décadas una ampliación de las luchas políticas. Según Weeks (1998), esto porque se han abierto a otras dimensiones no sólo identitarias sino en relación a derechos sociales como la salud, trabajo, educación o ámbitos civiles, pero también porque se enmarca en una disputa por el reconocimiento de otros valores, preferencias y placeres. A partir de los 90 se comienzan a ver estudios que cuestionan la idea de ciudadanía en las democracias liberales configurada en términos heteronormativos (EVANS, 1993; RICHARDSON, 2000; PLUMMER, 2001); es decir denunciar críticamente como en éstas los sujetos heterosexuales cuentan con mayores derechos y privilegios ciudadanos que aquellos sujetos no-heterosexuales. Así, se ha replanteado el significado del sexo, género y la sexualidad en relación a cómo se vinculan con los estados-nación modernos (LIND y ARGÜELLO, 2009). Lejos de mantenerse confinadas en la esfera privada, las cuestiones sexuales quedan sometidas cada vez más a las mismas exigencias políticas que el resto de las cuestiones de la sociedad: en nombre de los mismos valores de libertad e igualdad se interpela todavía más a las normas del género y la sexualidad (FASIN, 2012). Aunque el respeto por la diversidad sexual en Chile se ha posicionado como un valor democrático que determina la acción del Estado en torno al tema (GALAZ VALDERRAMA;

3 Esta normativa surge desde el trabajo intersectorial realizado por la sociedad civil, desde organizaciones LGBTI; equipos de establecimientos educacionales; académicos/as, el Instituto Nacional de Derechos Humanos (INDH), la Organización de las Naciones Unidas para la Educación, la Ciencia y la Cultura (UNESCO) y profesionales del Ministerio de Educación y de la Superintendencia de Educación. (MINEDUC, 2017). 
SEPULVEDA GALEAS; POBLETE MELIS; TRONCOSO PÉREZ; MORRISON JARA, 2018), en el ámbito educativo hay una persistencia de ideas de normalidad y estandarización, que tienen una expresión en la validación de hegemonías heterosexistas (RAMÍREZ y MENA, 2014). Hablar de diversidad sexual tiene "implicancias problemáticas ya que reproduce una idea de 'otredad' en tanto diferencia, reificando un ideal heteronormativo de normalidad" (GALAZ VALDERRAMA; SEPULVEDA GALEAS; POBLETE MELIS; TRONCOSO PÉREZ; MORRISON JARA, 2018, p. 2).

Las evidencias al respecto en el ámbito educativo (RÍOS, 2011; NÚÑEZ, 2013; BLANDÓN, 2014 y RAMÍREZ, 2017) muestran que quienes no responden a los patrones heteronormativos sufren episodios de violencia y exclusión, primando una visión masculinista-hegemónica que transmite valores de igualdad y libertad dentro de un modelo jerárquico, patriarcal y androcéntrico (RAMÍREZ y MENA, 2014, p. 112). Bolivar (2004) sostiene que la educación se ha erigido sobre la base de subordinar las identidades de diversos grupos al proyecto de creación de una ciudadanía universal, la que resulta restringida en el contexto de las sociedades contemporáneas. Como plantea Morgade (2011), toda educación es "educación sexual" y su sentido principal es preservar una parte importante del orden social de género establecido. Por lo tanto, se puede plantear que la educación sexual se ubica en un campo de disputa política, al entenderse como un dispositivo que establece límites entre lo que es aceptable para la sociedad y lo que queda fuera en las prácticas sexuales de la ciudadanía. Entonces la sexualidad ya no se presenta como tabú sino con ciertos límites de comprensión, haciendo que los ámbitos educativos funcionen como tecnologías de gobierno, que actúan como aparatos ideológicos (MORGADE, 2011; NAVA y LÓPEZ, 2010) respecto a establecer las buenas y correctas sexualidades. La educación sexual se constituye como un espacio instituido - ya que coloca los límites de lo considerado como normal - pero a la vez instituye posibilidades de construcción de subjetividades que se fugan de estos parámetros normativos, porque "en términos de relaciones de género y sexualidades, la educación formal, en forma contradictoria, silencia pero a la vez es un espacio de performance de los cuerpos sexuados" (MORGADE, 2011, p.28)

\section{Aspectos metodológicos}

Presentamos el análisis de un corpus textual configurado a partir de normativas educativas chilenas en el periodo postdictadura. Se hizo un barrido desde 
1990 a 2017, identificando documentos públicos que contuvieran temáticas vinculadas a la "diversidad sexual", además de los programas de educación sexual implementados por los gobiernos. Siguiendo lo señalado por Carrasco, López y Estay (2012), empleamos la indagación y el análisis documental para identificar dentro de los textos, categorías discursivas que fuesen significativas para identificar las formas de comprensión de la "diversidad sexual". Posteriormente, rastreamos ciertos repertorios interpretativos (WETHERELL y POTTER, 1996) en la construcción de esas otredades sexualidades.

\section{TABLA 1: DOCUMENTOS ANALIZADOS}

\begin{tabular}{|l|l|l|}
\hline Tipo & Contenido & Año \\
\hline Normativa & $\begin{array}{l}\text { Política de educación sexual para el mejoramiento de la } \\
\text { calidad de la educación }\end{array}$ & 1991 \\
\hline Política & $\begin{array}{l}\text { Política de educación en sexualidad para el mejoramiento de } \\
\text { la calidad de la educación (1993-2001) }\end{array}$ & 1993 \\
\hline Normativa & $\begin{array}{l}\text { Guía para las Jornadas de Conversación sobre Afectividad y } \\
\text { Sexualidades }\end{array}$ & 1999 \\
\hline Política & $\begin{array}{l}\text { Educación en sexualidad para el mejoramiento de la calidad } \\
\text { de la educación }\end{array}$ & 2003 \\
\hline Plan & $\begin{array}{l}\text { Plan nacional de Educación en Sexualidad y Afectividad } \\
\text { (2005-2010) }\end{array}$ & 2005 \\
\hline Ley 20.370 & Ley General de Educación & 2009 \\
\hline Reglamento & Convivencia escolar & 2010 \\
\hline Ley 20.536 & Violencia Escolar & 2011 \\
\hline Reglamento & $\begin{array}{l}\text { Propuestas sobre perfeccionamiento docente sobre } \\
\text { programas de afectividad, sexualidad y género }\end{array}$ & 2011 \\
\hline Normativa & Educación en sexualidad, afectividad y género & 2015 \\
\hline Plan Nacional & Plan Nacional de Educación Sexual & 2015 \\
\hline Ley 20.854 & Inclusión escolar & 2015 \\
\hline Orientaciones & $\begin{array}{l}\text { Para la inclusión de las personas lesbianas, gays, bisexuales, } \\
\text { trans e intersex en el sistema educativo chileno }\end{array}$ & 2017 \\
\hline
\end{tabular}

FUENTE: Elaboración propia

\section{Discusión: Diversidad sexual, aquello innombrable}

Como señala Romero y García Dauder (2003), las posiciones de sujeto están vehiculizadas a partir de ciertas "saturaciones identitarias" de ciertas categorías es decir la sobre-visibilidad de ciertas identidades en desmedro de otras diferencias 
del sujeto; o bien por opacidad o invisibilidad de estas diferencias. Siguiendo esta noción, veremos que, en las intervenciones públicas en Chile, la diversidad sexual se ha instalado preferentemente bajo una lógica de opacidad, transitando en los últimos seis años a cierta saturación de sólo algunas identidades no heterosexuales. Pareciera dominar la evitación de la enunciación explícita de las sexualidades, para que, a través de términos más generales, se posicione de forma implícita. Es decir, hablar de las sexualidades sin nombrarlas. La opacidad de las sexualidades emerge de soslayo en leyes y normativas que centran su análisis en la lógica de la inclusión y en el combate a la discriminación dentro de las escuelas bajo una perspectiva de derechos. Lo universal prima sobre lo específico sexual. Si bien en su momento algunos sectores sociales promovieron el uso explícito de la "diversidad u orientación sexual" en estas Les, finalmente en una disputa cultural terminó por imponerse dominantemente una lógica de opacidad de las identidades sexualizadas: en estos corpus normativos se evita establecer la referencia directa a estas otredades. Se apela a principios de no discriminación de manera universal, y de respeto a la "diversidad social" o "cultural" - como la ley general de educación y la ley de convivencia escolar. Pareciera que los tópicos generalistas como "social" o "cultural" englobaran cualquier tipo de discriminación y no fuera necesario explicitar las identidades sexuales. En esta argumentación, al quedar difuminada la diferencia sexual también quedan invisibles las desigualdades que puede padecer sujetos específicos por su identidad y/u orientación sexual en los espacios educativos. En el siguiente extracto de esta discusión de ley queda explícita esta opacidad en la que se mueve la concepción de diversidad sexual:

La Ministra de Educación hizo presente que en el artículo $2^{\circ}$ se consagra el principio de la diversidad, a través del cual se promueve el respeto a la diversidad cultural, religiosa y social y se prohíbe efectuar cualquier tipo de discriminación arbitraria. En votación la indicación número 198 (inclusión de la diversidad sexual), fue rechazada (...) (LEY 20.370, p. 704).

La ley de inclusión escolar (20.845/15) reinstala la noción del respeto a "la diversidad" en genérico, entendida como un todo "cultural y social" que debe promover el sistema público. En esa configuración generalista coexiste una multiplicidad de otredades, entre ellas las sexuales, que vuelve opaco las distinciones de su constitución. Asimismo, esta enunciación de una "diversidad opaca" se vincula a los principios de autonomía de cada centro educativo, dejando en el territorio de la decisión liberal, la posibilidad de concreción de estas diversidades. En la normativa se especifica que se entenderá como 
Diversidad. El sistema debe promover y respetar la diversidad de procesos y proyectos educativos institucionales, así como la diversidad cultural, religiosa y social de las familias que han elegido un proyecto diverso y determinado, y que son atendidas por él, en conformidad a la Constitución (...). El sistema debe permitir la adecuación del proceso a la diversidad de realidades, asegurando la libertad de enseñanza y la posibilidad de existencia de proyectos educativos institucionales diversos. (LEY 20.845, 2015, p.1)

A través de los distintos períodos políticos, se evidencian esfuerzos tímidos por dar visibilidad a otras identidades sexo-genéricas. En la "Política de Educación en Sexualidad para el Mejoramiento de la Calidad de la Educación” (1993) se señala que existen "actitudes diversas frente a la sexualidad, lo cual parece indicar que es necesario evitar generalizaciones, más bien, reconocer las particularidades que presenta este fenómeno y diferenciar situaciones a fin de abordar adecuadamente la formación de niños y jóvenes” (MINEDUC, 2001, p. 9).

En este documento se instala una visión de las relaciones sexuales en una lógica de derechos, pero reproduciendo la relación ineludible entre sexualidades e ideas axiológicas como el respeto, el amor y la responsabilidad en la práctica sexual. Si bien, se abre por primera vez a una cierta concepción de identidad sexual, a la vez concibe la sexualidad como un proceso de transmisión de saberes y vivencias por parte de los sistemas de socialización, donde nuevamente "la familia" heterosexual toma un rol primordial en el desarrollo de la identidad sexual.

Este juego entre evitación de la diversidad sexual y alusión generalista, también se visualiza en el reglamento de convivencia para las escuelas (2010). Pese a que socialmente se han incrementado los casos de lesbo, homo y transfobia en los espacios educativos (MOVILH, 2017), en el texto nuevamente se apela a la opacidad de las diferencias por el rescate explícito de una generalización en relación a un conjunto de diversidades contenidas en un único todo. El texto establece un reglamento "estándar" de convivencia a escolar, planteando entre sus objetivos:

promover y desarrollar en todos los integrantes de la comunidad educativa los principios y elementos que construyan una sana convivencia escolar... un derecho y un deber que tienen todos los miembros de la comunidad educativa, cuyo fundamento principal es la dignidad de las personas y el respeto que éstas se deben. Es un aprendizaje en sí mismo que contribuye a un proceso educativo implementado en un ambiente tolerante y libre de violencia, orientado a que cada uno de sus miembros pueda desarrollar plenamente su personalidad, ejercer sus derechos... (MINEDUC, 2010). 
Se produce un cierto juego dicotómico entre invisibilidad/visibilidad LGTBI: las encuestas sobre violencia, acoso o clima escolar llevadas a cabo por el Ministerio de Educación y la fundación Todomejora (MINEDUC, 2011 y TODOMEJORA, 2016), dan cuenta de la existencia de este tipo de situaciones (burlas, insultos, amenazas, golpes), pero los motivos que las generan se señalan como un compilado de situaciones posibles (etnia, clase, fenotipia, orientación sexual). Pareciera, entonces, que el acoso y violencia asociado a la presencia de estudiantes LGTBI en la escuela queda subsumido en categorías universales de acoso, de manera general lo que, en el fondo, le restan importancia.

En general esta opacidad respecto de la diversidad sexual y la construcción de un todo unificado de lo "diverso" constituido por múltiples otredades indiferenciadas, se verifica con más consistencia entre 1990 y 2011. Posterior a esa fecha, en el marco tanto de las acciones colectivas LGTBI como también casos de delitos de odio de gran connotación pública, como la muerte del joven Daniel Zamudio en 2012, se comienzan a visualizar ciertas "saturaciones identitarias" (ROMERO y GARCÍA DAUDER, 2003) respecto del reconocimiento de los sujetos LGTBI. La Ley anti-discriminación, la ley de Acuerdo de Unión Civil, la incorporación de elementos anti-discriminatorios en normativas en salud, en las fuerzas armadas, en el registro civil y la normativa laboral son parte del escenario contextual, donde además en el sector educativo comienzan a situarse discursivamente los sujetos LGTBI por cierto exceso identitario. Esto llega a su punto máximo con la comunidad transexual y transgénero en el año 2017. Además de las diversas circulares normativas en otras carteras ministeriales, se llega al Ordinario 768 en el ámbito educativo que plantea los derechos de niñas, niños y estudiantes trans. Hasta ese año, no existe ningún ordinario explícito en educación en referencia directa de la inclusión de otras identidades u orientaciones sexuales - personas lesbianas, gays o bisexuales, pese a la denuncia constante de acoso homofóbico. Sin embargo, la niñez trans emerge en estas normativas como un sujeto problemático para el ámbito educativo, susceptible de intervención y como sujeto necesitado de esta actuación. Esto precisamente evidencia la lógica heteronormativa de la escuela chilena ya que la sola presencia de sujetos no binarios desafía los elementos de integración estandarizados concebidos como de inclusión de la "diversidad" de las escuelas y la lógica de derechos hasta esa fecha imperante. Con el Ordinario se instala, por tanto, la idea de que el problema es la falta de orientación en las escuelas frente a identidades específicas no mayoritarias y que, para evitar posiciones arbitrarias de los establecimientos, se requiere normar:

[...] la realidad de los niños, niñas y estudiantes trans, que emerge y se visibiliza cada vez más en los establecimientos educacionales, 
desafía a las comunidades a conocerla e incluirla con respecto en las gestiones y prácticas educativas. (ORDINARIO No 0768, 2017).

La lógica de derechos en la escuela, de esta manera, adquiere especificaciones respecto de la identidad sexual no binaria (personas trans), pero esa lógica de derechos cambia, se generaliza y vuelve a una noción abstracta, respecto de las orientaciones sexuales (personas lesbianas, homosexuales, bisexuales). Cabe señalar que el interés creciente del Estado por la incorporación de una educación sexual ha tenido énfasis distintos de acuerdo al marco valórico de los gobiernos de turno. Esta incorporación la caracterizamos bajo tres aristas en relación a las sexualidades: por un lado, con límites heteronormativos y binarios a la hora de considerar el sexo y el género (hombre/mujer o bien, homosexualidad/ heterosexualidad), por otro, con la legitimización de saberes biologicistas que fundamentan preferentemente intervenciones de prevención de riesgos (sobre todo embarazo adolescente y de enfermedades de transmisión sexual), y por último, condicionado a discursos axiológicos que establecen el horizonte ético de lo que es o no es una correcta relación sexual dentro de la sociedad chilena.

Aunque esta incorporación creciente de las sexualidades se relaciona con el marco internacional de derechos, a la vez se consagra en su vertiente más tradicional, a partir de su relación con los principios establecidos en la Constitución política de Chile - promulgada en dictadura en 1980 - donde la "familia heterosexual" emerge como pilar social. En efecto, se ha mantenido una visión binaria de las sexualidades (hombre-mujer), vinculando los discursos de la sexualidad a las nociones de familia y responsabilidad e invisibilizando las sexualidades no heterosexuales.

Así se denota en las recomendaciones iniciales de la comisión consultiva en Educación Sexual en el primer gobierno democrático, donde la sexualidad mantiene la lógica imperante en la dictadura, instalándose en una dimensión binaria y normativa, vinculándose a la "reproducción", a la idea de "familia" (MINEDUC, 1991, p.16). Posteriormente en el programa público JOCAS, entre 1996 y 2000, se ponen como centro, por un lado, la idea de "sexualidad responsable" (donde la responsabilidad se relaciona al cuidado de enfermedades e infecciones de transmisión sexual), y por otro, el compromiso en relación a "la familia heterosexual" como entidad superior a la comprensión de la propia sexualidad individual. Se invisibiliza, en este marco explicativo, temáticas relacionadas con la diversidad sexual, y aunque se habla de respeto a distintas orientaciones sexuales y de la capacidad de discernimiento individual, se da énfasis al compromiso familiar futuro y procreativo, y la idea de matrimonio heteronormativo. En el plan de educación sexual de 2015, se enfatiza que la 
sexualidad debe ser "sana, plena y responsable", excluyendo en ello otras temáticas como puede ser el goce, autoconocimiento sexual, experiencias no heterosexuales, entre otras. Esta argumentación se mantiene en las bases curriculares de la asignatura de Orientación de séptimo a segundo medio (2016), en que se reifica el vínculo entre sexualidad y afectividad. Así se enfatiza que:

educar para una sexualidad sana y responsable significa formar personas reflexivas, dialogantes y tolerantes, capaces de identificar conductas de riesgo y de elegir cursos de acción, anticipando las consecuencias de sus acciones, para sí y los demás. Significa, en definitiva, formar personas conscientes de su individuación y dispuestas a generar modos de relación respetuosos en las comunidades (MINEDUC, 2016).

En los entramados discursivos de la educación sexual del Plan Ministerial de Educación Sexual del año 2015, se establece un nexo inviolable entre sexo y afectividad, situando como nudo problemático exclusivamente la falta de información, la prevención de enfermedades y embarazo, y la importancia de la conciencia de una sexualidad "responsable". No sólo se individualiza en el sujeto "estudiante" el foco de la responsabilidad sobre la sexualidad, además de centrar la sexualidad al acto coital y sus consecuencias, sino que además se presupone que es un tema de disputa entre el ámbito educativo y familiar, destacando constantemente la libertad de conciencia, valores y convicciones familiares. En ese sentido, aunque se plantea una preocupación del Estado, a la vez se deja entrever que, en un marco liberal como el chileno, el Estado tiene límites para incorporar consistentemente esta temática. Se deja en autonomía a los establecimientos educacionales, la incorporación progresiva de estas temáticas, muchas veces terciarizando en apoyos educativos de la sociedad civil $(\mathrm{ONG})$, de las universidades o bien, entidades religiosas ${ }^{4}$.

Es importante visibilizar la transversalidad de contenidos biologicistas enfocados en la prevención de ETS e ITS, pero además tener en cuenta que la

4 Una de las primeras entidades en ofrecer formación en educación sexual fue CEMERA en los 90. Dentro de sus conceptos básicos recalcaba que "en la pubertad, junto con estos cambios, aparece el interés por el sexo opuesto y se adquiere la capacidad reproductiva" (CEMERA,1993, p.24). Es decir, concebía las relaciones sexuales entre hombre y mujer, promoviendo sólo este tipo de relaciones interpersonales, excluyendo e invisibilizando a las parejas del mismo sexo, intersexualidad y bisexualidad. Su enfoque destaca que "nacemos y morimos con un sexo determinado, hombre o mujer, y nos diferenciamos anatómicamente entre los sexos" (CEMERA, 1993, p.44), dejando explícita la perspectiva biologicista y la naturalización de identidades sexuales fijas. 
ley habla que debe incorporarse este tipo de formación desde enseñanza media, es decir, sobre los 14 años. Esto muestra un sesgo por edad, sin considerar que los sujetos están inmersos a inquietudes sexuales desde la niñez. De esta manera, la comprensión de sexualidad que transmite el corpus normativo, se basa en situarla en un marco de edad (exclusividad para jóvenes y personas adultas), y relacionada casi exclusivamente al acto del coito, más que desde otras experiencias sensibles como el placer, el conocimiento del cuerpo y las relaciones, la afectividad, la orientación e identidad sexual, entre otros aspectos (GALAZ VALDERRAMA; SEPULVEDA GALEAS; POBLETE MELIS; TRONCOSO PÉREZ; MORRISON JARA, 2018).

Con la terciarización de servicios formativos de educación sexual en el primer gobierno de derecha post-dictadura y que se afianza a través de los años, se polariza la formación sexual y la apertura a temáticas de diversidad sexual en el país. Esto lleva a posicionar el tema de la educación sexual como un campo de disputa respecto de la concepción de sexualidad normativa y no normativa, entre sectores progresistas y conservadores de la sociedad chilena. Se visualiza una polarización de concepciones frente a la educación sexual, en que los colectivos LGTBI emergen como elementos extraños y/o patológicos frente a visiones más abiertas de estos colectivos. Mientras, una parte optó por programas más integrales de educación sexual ${ }^{5}$, también un sector importante lo hizo por planes de carácter tradicional, especialmente vinculada a una formación religiosa, que en algunos casos vinculó homosexualidad a un "trastorno de identidad sexual" 6 (PALMA, REYES y MORENO, 2013). Entre 1990 al 2005, el rol de Estado estuvo centrado en la prevención de enfermedades de transmisión sexual y combate al embarazo adolescente que a otros aspectos de la educación sexual (TRONCOSO, GALAZ y MORRISON, 2016). Años posteriores a la promul-

5 Algunas escuelas entre municipales y particulares subvencionados, optaron en el periodo por el Programa de Aprendizaje, Sexualidad y Afectividad (PASA), de la Facultad de Psicología de la Universidad de Chile, el cual pone de relieve la igualdad de género, la no discriminación a las personas por su orientación sexual y métodos de prevención. Otros establecimientos optaron por la Asociación Chilena de Protección de la Familia, Sexualidad, autoestima y prevención del embarazo en la adolescencia (APROFA), el cual está enfocado en el derecho a la salud sexual y reproductiva, y que incluye orientación en diversidad sexual, homosexualidad, VIH, anticonceptivos y autoestima.

6 Entre éstos se cuentan el programa de Educación en Valores, Afectividad y Sexualidad (PAS) desde la Pontificia Universidad Católica de Chile, el programa Teen Star, de la facultad de Ciencias biológicas; en los que se plantearon como ejes temáticos el amor, la educación de la voluntad ante el deseo sexual y los métodos de planificación familiar. El foco central giraba en la conformación de parejas heterosexuales, enfatizando el control de la reproducción, la selección responsable de la pareja y el fortalecimiento de la familia tradicional. También el programa "Aprendiendo a querer" de la Universidad de la Santísima Concepción, que centra sus objetivos en la valorización de la abstinencia, el matrimonio exclusivo entre hombre y mujer y la defensa de los roles tradicionales de género. 
gación de ley anti-discriminación (2012) y la visibilidad desde el 2014 de entidades de familias de niños/as trans, la opacidad entorno a la diversidad sexual comienza a perder peso y se enuncia explícitamente la necesaria formación en relación a la identidad y orientación sexual. No obstante, con la terciarización liberal de servicios educativos en educación sexual, finalmente se producen diferencias significativas entre los y las adolescentes en cuanto a su formación. Esta "asimetría formativa" que deriva en la generación de brechas actitudinales y de conocimiento entre los y las estudiantes del país constituye una forma más de la desigualdad, esta vez amparada bajo la idea de la libertad de elección de las familias promovida por el Estado.

\section{Conclusiones}

A través del análisis vemos que un aspecto relevante que surge es la insistencia del Estado de situarse en una perspectiva de derechos con características universales como repertorio interpretativo; noción sobre la cual funda su legitimidad. En efecto, en las normativas analizadas hay una alusión reiterativa a los derechos humanos como paradigma fundante y horizonte común que justifica las intervenciones públicas, moviéndose entre una lógica antidiscriminatoria y de derechos sociales (WEEKS, 1998). Sin embargo, la posibilidad de repensar y cuestionar una relación entre derechos humanos y una ciudadanía mayoritaria heteronormada, sólo emerge tímidamente a partir del año 2012. En la consolidación democrática, persiste una visión restrictiva de derechos, ligada a los sujetos individuales, cuyo derecho ciudadano se configura en términos heteronormativos (EVANS, 1993; RICHARDSON, 2000; PLUMMER, 2001), contando con mayores derechos y privilegios que aquellos sujetos no heterosexuales. No obstante, no se puede desconocer que, en el tránsito democrático chileno, la sexualidad ha sido un tema que ha pasado a formar parte del repertorio político. Al decir de Sabsay (2016), la noción de diversidad sexual tiene lugar en los procesos de democratización sexual, lo cual puede ser considerado como un avance, sin embargo, lo relevante es indagar en la forma en que ocupa el campo político, toda vez que es un ámbito en disputa espacio a una concepción heteronormativa. En ese sentido, vemos con el análisis que en Chile la educación sexual binaria emerge como una forma de control al hacer aparecer a ciertas sexualidades preferentes (heterosexuales) e invisibilizar otras posibilidades (sexualidades no binarias). Creemos que las directrices en la educación pública subordinan identidades a un proyecto mayor, que dice relación con la creación de una iden- 
tidad nacional universalista que prioriza una visión hetero-céntrica, y que admite como única posibilidad frente a la diversidad, la asimilación. Por lo mismo, acciones como el acoso hacia colectivos y grupos LGTBI muchas veces quedan invisibles, ante una actuación pública que atiende a la generalidad del acoso escolar, sin apellido. Pese a ello, existe cierta emergencia de ciertas sexualidades no heterosexuales lo que va abriendo luces de reconocimiento (BUTLER, 2009) aunque siempre se establece en relación a una vieja dicotomía: mayoría/ minorías (heterosexuales/otredad no heterosexual). En síntesis, en este análisis de las políticas educativas post-dictadura en Chile, vemos que la diversidad sexual tiene una doble lectura: por un lado, a través de ella se busca atenuar desigualdades y promover derechos - como está sucediendo en los últimos años al abrir la posibilidad de reconocimiento de niños/as trans; pero, por el otro, al enunciarla como un todo generalista, desdibujando sus específicas desigualdades y violencias, se transforma en una forma más de un poder disciplinador que se ejerce sobre los individuos y sus cuerpos (FOUCAULT, 1977).

\section{REFERENCIAS}

ARENAS, L.; DURAN, J.; DIDES, C.; FERNÁNDEZ, C. Primer Informe Salud Sexual Salud Reproductiva y Derechos Humanos En Chile. Santiago: Corporación Miles, 2016.

BOLIVAR, A. Ciudadanía y Escuela Pública en el Contexto de Diversidad Cultural. Revista Mexicana de Investigación Educativa, México, v. 9, n. 20, p. 15-38, 2004.

BUTLER, J. Performatividad, precariedad y políticas sexuales. Revista de Antropología Iberoamericana, Madrid, v.4, n.3, 321-336, 2009.

CAREAGA, G. Sexualidades diversas. Aproximaciones para su análisis. México: PUEG/UNAM, 2004.

CARRASCO, C.; LÓPEZ, V.; ESTAY, C. Análisis crítico de la Ley de Violencia Escolar de Chile. Psicoperspectivas, Valparaíso, v. 11, n. 2, 31-55, 2012.

EVANS, D. Sexual Citizenship: The Material Construction of Sexualities. London: Routledge, 1993.

FASSIN, E. La democracia sexual y el choque de civilizaciones. Mora, México, 18, 5-10, 2012.

FOUCAULT, M. Historia de la sexualidad I: La voluntad de saber. Madrid: Siglo XXI, 1977. 
GALAZ VALDERRAMA, C.; SEPULVEDA GALEAS, M.; POBLETE MELIS, R.; TRONCOSO PÉREZ, L.; MORRISON JARA, R. Derechos LGTBI en Chile: Tensiones en la constitución de otredades sexualizadas. Psicoperspectivas, Valparaíso, v. 17, n. 1, p. 1-11, 2018. <https://doi.org/10.5027/psicoperspectivas-vol17-issue1-fulltext-1165>.

GONZÁlEZ, M.; NÚÑEZ, C. G.; GALAZ VALDERRAMA, C.; TRONCOSO, L.; MORRISON JARA, R. Editorial Sección Temática: Los usos de la diversidad sexual en la acción pública. Revista Psicoperspectivas, Valparaíso, v. 17, n. 1, p. 1-6, 2018. $<$ https://dx.doi.org/10.5027/psicoperspectivas-vol17-issue1-fulltext-1315>.

LIND, A.; ARGÜELLO, S. Ciudadanías y Sexualidades en América Latina. Presentación del Dossier. Iconos: Revista de Ciencias Sociales, Quito, n. 35, p. 13-18, 2009.

LEY N $^{\circ} 20.370$ Ley General de la Educaciòn. Ministerio de Educación. 2009. Consulta en: <https://especial.mineduc.cl/wp-content/uploads/sites/31/2018/04/LEY-20370_12SEP-2009.pdf $>$.

LEY N $N^{\circ} 20.845$ de inclusión escolar. Ministerio de Educación. 2015. Consulta en: $<$ https://leyinclusion.mineduc.cl/>.

MINEDUC. Hacia una política de educación sexual para el mejoramiento de la calidad de la educación. Ministerio de Educación. Santiago: Gobierno de Chile, 1991.

MINEDUC. Política de Educación en sexualidad para el mejoramiento de la calidad de la educación. Ministerio de Educación. Santiago: Gobierno de Chile. 2001.

MINEDUC. Plan nacional de Educación en Sexualidad y Afectividad (2005-2010). Ministerio de Educación. Santiago: Gobierno de Chile. 2005.

MINEDUC. Programa en sexualidad, afectividad y género. Ministerio de Educación. Santiago: Gobierno de Chile. 2015. Consulta disponible en: $<$ http://www.convivenciaescolar.cl/usuarios/convivencia_escolar/File/2018/sexualidad-nov.pdf $>$. Acceso en: 24 de septiembre de 2018 .

MINEDUC. Reglamento de convivencia escolar. Ministerio de Educación. Santiago: Gobierno de Chile, 2010

MINEDUC. Encuesta sobre violencia, acoso o clima escolar. Ministerio de Educación. Santiago: Gobierno de Chile. 2011. Consulta en: <http://www.convivenciaescolar.cl/ usuarios/convivencia_escolar/File/2014/PrevenciOnagresiOnyacoso2011.pdf >.Acceso en: 24 de septiembre de 2018 .

MINEDUC. Bases curriculares asignaturas de orientación de séptimo a segundo medio. Ministerio de Educación. Santiago: Gobierno de Chile. 2016.

MORGADE, G. Toda educación es sexual: Hacia una educación sexuada justa. Buenos Aires: La Crujía Ediciones, 2011.

MOVILH. XIII Informe Anual: Derechos Humanos de la Diversidad Sexual Chilena. Santiago: MOVILH, 2015. 
MOVILH. XIII Informe Anual: Derechos Humanos de la Diversidad Sexual Chilena. Santiago: MOVILH, 2017.

NAVA, D.; LÓPEZ, G. Educación y discriminación de género. El sexismo en la escuela primaria, un estudio de caso en Ciudad Juárez. México D.F: UAM-I/Ediciones Eón/ CIESAS/CONACYT, 2010.

NÚÑEZ, Y. Discriminación basada en la orientación sexual e identidad de género no normativas hacia jóvenes en el sistema educativo Chileno. Santiago: Universidad Alberto Hurtado, 2013.

ORDINARIO No 0768. Derechos de niñas, niños y estudiantes trans en el ámbito de la educación. Ministerio de Educación, 2017.

PALMA, I.; REYES, D.; MORENO, C. Educación sexual en Chile: Pluralismo y libertad de elección que esconde una propuesta gubernamental conservadora. Revista Docencia, Santiago, n. 48, p. 14-24, 2013.

PLUMMER, K. The Square of Intimate Citizenship: Some Preliminary Proposals. Citizenship Studies, London, v. 5, n. 3, p. 237-253, 2001.

RAMÍREZ, F. Inclusión educativa de estudiantes sexualmente diversos en Colombia periodo 2000-2015. Comprensiones pedagógicas de las sentencias de la Corte Constitucional. 2017. Tesis (Doctor en Educación) - Universidad Santo Tomás, Bogotá, 2017.

RAMÍREZ, F.; MENA, M. Aportes desde la perspectiva queer para la reforma curricular de la escuela en la búsqueda de la equidad de género. Revista Ciudad Paz-ando, Bogotá, v. 7, n. 1, p. 106-124, 2014.

RICHARDSON, D. Constructing Sexual Citizenship: Theorizing Sexual Rights. Critical Social Policy, London, v. 20, n.1, p. 105-135, 2000.

RÍOS, J. La homofobia en las escuelas: implicaciones para el desarrollo del líder GLBT. Puerto Rico, Gurabo: Universidad de Turabo, 2011.

ROBLES, V. H. Bandera Hueca. Historia del movimiento homosexual de Chile. Santiago, Chile: ARCIS/Cuarto Propio, 2008.

ROMERO, C.; GARCÍA DAUDER, S. Saturaciones identitarias: de excesos, materialidades, significación y sus (in) visibilidades. Revista CLEPSYDRA, Madrid, n. 2, p. 37-56, 2003.

SABSAY, L. Fronteras sexuales: Espacio urbano, cuerpos y ciudadanía. Buenos Aires: Paidós, 2011.

SABSAY, L. The political imaginary of sexual freedom: Subjectivity and power in the new sexual democratic turn. Basingstoke, UK: Palgrave Macmilan, 2016.

SAWAIA, B. Psicología y desigualdad social: una reflexión sobre libertad y transformación social. Psicologia \& Sociedade, Minas Gerais, v. 21, n. 3, p. 364-372, 2002. 
TODOMEJORA. Encuesta sobre violencia, acoso o clima escolar. Todomejora. 2016. Consulta en: $<$ https://todomejora.org/wp-content/uploads/2016/08/Encuesta-de-Clima-Escolar-2016-Fundacion-TODO-MEJORA.pdf $>$.

TRONCOSO, L.; GALAZ, C. y MORRISON, R. Miradas Críticas sobre la Intervención Educativa en Diversidad Sexual. Revista Latinoamericana de Educación Inclusiva, Santiago de Chile, v. 10, n. 2, p. 93-111, 2016.

VALENTINE, G. Theorizing and Researching Intersectionality: A Challenge for Feminist Geography. The Professional Geographer, Minesota, v. 59, n. 1, p. 10-21, 2007.

WEEKS, J. The sexual citizen. Theory, Culture and Society, London, v. 15, n. 3-4, p. 35-52, 1998.

WETHERELL, M.; POTTER, J. El análisis del discurso y la identificación de los repertorios interpretativos. Psicologías, Discursos y Poder, Madrid, n. 66, p. 63-78, 1996.

Texto recibido el 31 de octubre de 2018. Texto aprobado el 23 de enero de 2019. 